\title{
"Graziella et les trois éléments de l'univers dans "Graziella" de Lamartine"
}

\author{
Par \\ Noha Abdel Aziz Rizq* \\ noha.mabrouk@art.aun.edu.eg
}

\section{Résumé}

Lamartine était à la fois prosateur et poète et même un homme de politique. Après la mort de sa mère, Lamartine respire-t-il une autre atmosphère qui agit d'une puissance sur son atmosphère qui agit d'une puissance sur son esprit comme sur son âme. En 1811, un premier voyage en Italie l'a beaucoup enchanté. C'est là qu'il a rencontré Graziella, l'hérö̈ne métamorphosée, très idéalisée, d'un roman unique que Lamartine n'a écrit qu'à l'âge de cinquante-trois ans. On peut dire que Graziella est un chefd'œuvre du cœur et en même temps du style. Dans ce roman, admirablement composé, Lamartine ravit l'esprit par son style bien imagé qui fait vivre le lecteur dans un état constant d'ivresse. Si Lamartine a excellé dans sa poésie, il a excellé aussi dans ses écrits en prose et tout spécialement dans Graziella. C'est pourquoi nous pouvons dire que Lamartine est à la fois un grand poète et un grand écrivain en prose. Notre étude s'intéresse tout particulièrement à analyser le caractère du personnage principal Graziella qui constitue le titre de l'ouvrage romanesque et ensuite nous étudierons les trois éléments dominants dans ce roman : la nature, l'amour et la religion. Finalement, nous essayons d'examiner la variété stylistique.

Mots clés: Lamartine - Graziella - La nature - L'amour - La religion

\footnotetext{
* Professeur adjoint, Département de Français, Faculté des Lettres, Université d'Assiout
}

(Graziella et les trois éléments de l'univers ...) Dr. Noha Abdel Aziz Rizq 
Alphonse de Lamartine est l'une des figures très apparentes des poètes et des écrivains du $\mathrm{XIX}^{\mathrm{e}}$ siècle. II constitue avec Victor Hugo, Alfred de Musset, Baudelaire et plusieurs auteurs une espèce de constellation qui crée l'âme de la poésie et de la prose romantiques au $\mathrm{XIX}^{\mathrm{e}}$ siècle. En effet, les écrivains de ce siècle ont poussé la pensée et les sentiments vers des horizons nouveaux qui distinguent ce siècle que plusieurs critiques littéraires et artistiques appellent, à juste titre le grand siècle.

Ce qui est vraiment curieux, c'est que la plupart des écrivains de ce siècle romantique, réaliste et même naturaliste, ont écrit dans des domaines variés: littérature, critique, poétique, philosophique ...etc. Ils sont à la fois poètes, romanciers, et penseurs. Comme par exemple Lamartine.

Si Lamartine a excellé dans sa poésie, il a excellé aussi dans ses écrits en prose et tout spécialement dans Graziella que nous étudions dans cet article.

Graziella est un chef-d'œuvre de Lamartine. C'est un roman où l'écrivain a réussi à charmer les lecteurs par la description de la nature et par la perfection du style. C'est d'après Maurice Toesca "la meilleure chose que Lamartine ait faite en prose" ${ }^{1}$. 
Dans ce roman, admirablement composé, Lamartine ravit l'esprit par son style bien imagé qui fait vivre le lecteur dans un état constant d'ivresse. II poursuit son analyse dans des termes qui s'appliquent parfaitement à son existence au milieu de la nature charmante.

En 1823, Lamartine a publié Les Nouvelles Méditations et Les Harmonies poétiques et religieuses en 1830. Cependant, après les excès de la démagogie qu'il avait déchirée, Lamartine était sur le point de perdre sa popularité et devait rentrer dans la vie privée. La poésie lui est restée sa seule occupation et lui a causé parfois une espèce de mélancolie. Pour cela il a écrit Graziella pour garder sa popularité.

Ce roman se distingue par une facilité coulante des mots et des phrases. En effet, Graziella est plein de douceur et d'harmonie comme sa poésie. C'est pourquoi nous pouvons dire que Lamartine est à la fois un grand poète et un grand écrivain en prose $^{2}$.

Nous trouvons que le roman de Graziella est la plus littéraire des ouvrages de Lamartine, selon plusieurs critiques comme De Luppé qui dit que ce roman a fait beaucoup pour la 
gloire de son auteur et qu'il " est resté à coup sûr son œuvre la plus répandue, la plus lue aujourd'hui encore peut être" 3

Notre étude s'intéresse tout particulièrement à analyser le caractère du personnage principal Graziella qui constitue le titre de l'ouvrage romanesque. Nous essayons de connaître qui était Graziella ? L'auteur n'a pas rependu à cette question. Ensuite nous étudierons les trois éléments dominants dans ce roman : la nature, l'amour et la religion. En fin, nous essayons d'examiner la variété stylistique dans le roman.

\section{La peinture de l'héroïne :-}

Avant de parler de ce personnage, on trouve nécessaire de jeter quelque lumière sur son origine. Qui était Graziella ? On ne sait pas si elle s'appelle réellement Graziella ou non. Est-ce que ce nom est dérivé du diminutif du pronom de la mère de Lamartine Grazia Maria ${ }^{4}$ ? Ou d'Antoniella ou Antoine appelée aussi Elvira ${ }^{5}$ qu'il a rencontré à I'Italie.

Pour bien comprendre, il faut jeter quelque lumière de la vie de Lamartine (sa relation étroite avec sa mère, et son voyage à l'Italie).

Le père de Lamartine, le chevalier de Lamartine de part, ancien officier a remis à sa femme le soin et le souci de élever son fils 
- Cette mère, profondément croyante, mais sans étroitesse d'esprit et de pensée, délicate, romanesque, sensible, a réussi à bien former l'âme de son fils unique qu'elle adorait.

En effet, l'éducation que Lamartine recevait de sa mère a joué un grand rôle dans la formation de son esprit, son imagination et sa conception de la vie toute entière. Lamartine lui-même a déclaré l'influence de cette conception maternelle selon ce que dit J. J. Lucas Duberton dans son livre intitulé Lamartine :

"Mon éducation était toute dans les yeux plus ou moins serein et dans le sourire plus ou moins ouvert de ma mère. Les rêves de mon cœur étaient dans le sien... Je ne suis qu'un misérable après de ma mère" 6 C'était elle qui suivait le développement de ses sentiments et ses idées. II a trouvé, chez sa mère, des accents profonds lorsqu'il l'a perdue :

"le bord de sa robe était mon horizon"7

Aussi, Lamartine respire-t-il une autre atmosphère qui agit d'une puissance sur son atmosphère qui agit d'une puissance sur son esprit comme sur son âme. En 1811, un premier voyage en Italie l'a beaucoup enchanté. Pour le poète 
"l'Italie n'est pas seulement une terre ; c'est un instrument de musique, c'est l'orgue du monde. II suffit qu'un sentiment souffle dans les âmes pour que tout y résonne" ${ }^{8}$. C'est là qu'il a rencontré Graziella, I'héroïne métamorphosée, très idéalisée, d'un roman unique que Lamartine n'a écrit qu'à l'âge de cinquante-trois ans.

En réalité, I'Italie a toujours joué un grand rôle dans la vie de Lamartine. II y a rencontré Elvire et l'a aimée. Elvire est morte brusquement et la douleur a ouvert dans l'âme de cet écrivain-poète, la source d'une poésie nouvelle. C'est de là qu'est sorti le recueil des Méditations qui a rencontré un grand succès.

En effet, Duberton rapporte dans son livre intitulé Lamartine que cet auteur "a l'art d'entasser les contre-vérités et qu'il donne la tablature, non seulement à la postérité, mais aussi à ses contemporains" ${ }^{9}$.

Dans ses mémoires, publiés après sa mort en 1871 , Lamartine raconte qu'il y avait deux jeunes filles chez son cousin Dareste de la Chavanne "l'une de vingt à vingt-cinq ans, Antoniella ... et son amie Graziella qui l'aide dans sa tâche et s'occupe aussi de la maison; elle a quatorze ans. La première était bien, mais sans rien de remarquable : la 
seconde était une ravissante jeune fille d'une beauté grecque. II l'aima bien vite, et lorsqu'il quitta Naples pour une excursion de quelques jours, elle s'enfuit en pleurs à procida. Elle serait donc morte de douleur cinq mois après son depart"10.

Cependant, dans une lettre à une personne appelée Virieu, Lamartine dit-comme le rapporte J. Lucas Duberton que "Graziella se serait appelée Antoniella ; plus âgée que son amant qui la nommerait sa mère. Elle serait morte d'une maladie de langueur,"11

On peut ainsi dire que Graziella est Antoniella. Elle a de vingt à vingt-cinq ans. De Luppe (Marquis) appruvie ce point de vue lorsqu'il rapporte qu'"elle seule a existé ... Ces vingt à vingt-cinq pourraient être l'âge véritable de la véritable Antoniella"12.

D'après le roman de Lamartine, Graziella appartient à une famille pauvre d'un vieux pêcheur qui s'appelle Andréa et qui avait "une espèce de honte de la pauvreté de sa demeure" ${ }^{13}$.

Graziella vivait dans une ambiance pauvre où elle et les membres de sa famille s'efforçaient d'aider le vieux pêcheur. Sa grand-mère vendait "les poissons devant (la) porte" ${ }^{14}$ de la 
maison ; Graziella restait "à la maison pour filer ou pour préparer les repas"15.

Mais cette fille laborieuse refusait de vivre dans cette pauvreté. Elle "apprenait un état bien au-dessus de l'humble profession de sa famille"16. Elle commençait à exercer un bon métier ; "Elle apprenait à travailler le corail" ${ }^{17}$ pour gagner sa vie et pour aider ses petits frères à vivre.

En effet, cette tâche pénible a formé le caractère très vif de Graziella. Elle était bien intelligente ; et ayant le goût du travail; elle "se perfectionnait dans son art" ${ }^{18}$. Son salaire lui permettait de tenir ses petits frères plus propres et mieux vêtus et de se donner à elle-même quelques costumes plus riches et plus élégants, comme c'était le cas des femmes de leur île.

C'est ainsi que Lamartine nous présente une fille laborieuse, charitable et d'une pitié extraordinaire dans ses relations avec les membres de sa famille. Notre poète-écrivain nous en donne un portrait bien vivant qui se compose de détails ne s'appliquant qu'au modèle. II a choisi les traits physiques et moraux les plus saillants et les plus propres à mettre en relief l'enchantement de Graziella. Dans ce portrait, on distingue "les cheveux longs et noirs (...) une taille élevée et mince (...), des yeux ovales et grandes"19 qui sont "d'une couleur 
indécise entre le noir foncé et le bleu de mer, qui adoucit le rayonnement par l'humidité du regard et qui mêle à proportions égales dans des yeux de femme la tendresse de l'âme avec l'énergie de la passion" ${ }^{20}$, des "joues pleines, arrondies, d'un contour ferme, mais d'un teint un peu pâle ..., non cette pâleur maladive du Nord, mais de cette blancheur saine du Midi"21.

Sa bouche aux lèvres ouvertes et épaisses, laisse voir des dents courtes et brillantes.

Elle était aussi une "belle fille du soleil et de la mer" ${ }^{22}$. Lamartine a réussi à nous faire sentir les "soleils de Procida (...) ruisseler de son front, de son corps et de ses pieds" 23 .

D'autre part, ses paroles, sa figure et sa physionomie expriment un caractère beau et doux. Sa voix enfantine est "claire et douce"24; elle a "une ravissante figure de jeune fille" 25 ; "Ses paroles (...) résonnaient comme une musique dans (les) oreilles. Sa physionomie est toujours changeante. Elle passe en une minute" ${ }^{26}$ de la surprise à l'effroi, de l'effroi à la gaieté, de la tendresse au rire ${ }^{27}$.

Aussi les gestes de Graziella reflètent-ils son caractère enfantin. Elle est une fille illettrée. Elle n'a jamais vu de livres qu'à l'église.

(Graziella et les trois éléments de l'univers ...) Dr. Noha Abdel Aziz Rizq 1043 
On constate aussi que Lamartine n'oublie pas de nous exprimer cette correspondance entre l'intérieur et l'extérieur, entre le côté physique et le côté moral de ce personnage. Elle se montre bien sensible. Après avoir entendu quelques pages de Paul et Virginie, "les teintes orageuses de la passion marbraient son front, le blanc azuré de ses yeux et de ses joues" 28 .

C'est une fille dont la physionomie est toujours le miroir de son âme ; et l'on peut savoir, d'après les traits de son visage, ses sentiments intérieurs.

Émue par Paul et Virginie, elle "n'avait pas dormi" et "son cœur était encore gros de chagrins" ${ }^{29}$.

Lamartine, en lisant Paul et Virginie à la jeune fille plus d'une fois, nous donne une documentation intéressante sur la mentalité de Graziella. C'est une fille rêveuse ; elle ne cherche pas la variété dans la poésie ou dans la musique comme c'est le cas du peuple du Midi. "On s'y nourrit, sans satiété (...) du même récit et du même air pendant des siecles" ${ }^{30}$.

Parfois, elle se montre gaie et folâtre ; elle "jouait de la guitare" ${ }^{31}$ et dansait la tarentelle.

Mais la qualité dominante de ce personnage est la tendresse et la pitié. Quand Lamartine est tombé malade après 
le départ de son ami Virieu, Graziella "jeta un cri de pitié (...); elle fit quelques pas en s'élançant vers (son) lit, puis, se retenant et s'arrêtant debout (...) la tête penchée sur l'épaule gauche dans l'attitude de la Pitié" 32 .

Elle se montre bien fidèle. Elle reproche à Virieu de partir et laisser son ami Lamartine tout seul en proie de sa maladie. Elle dit: "Ah ! si j'avais été à sa place, je ne serais pas partie, $\mathrm{moi}^{33}$.

Sa voix et ses yeux sont sereins. Elle a une attitude calme et une physionomie naïve ; et c'est ce qui adoucissait la souffrance de Lamartine.

Elle est aussi une fille vertueuse. Les visites des églises sont pour elle son "seul et pieux divertissement qu'elle connût et qu'elle aimât" ${ }^{34}$.

Elle se montre bien élégante. Son costume révèle souvent un aspect de son caractère : il la montre soigneuse, jolie, distinguée et polie : "des soubrevestes de soie rayée de noir et de vert : ces vestes galonnées sur les coutures flottent ouvertes sur les hanches, elles laissent apercevoir par devant la finesse de la taille et les contours du cou orné de colliers ; enfin de larges boucles d'oreilles ciselées où les fils d'or s'entrelacent avec de la poussière de perles" ${ }^{35}$. 
Elle est aussi bien soucieuse de la parure ; elle s'émaille de mettre "quelques fleurs de grenades rouges ou de lauriers-roses sur le côté de la tête dans ses cheveux noirs" ${ }^{\prime 36}$.

Elle est une "humble fille au cœur aimant" ${ }^{37}$. C'est ainsi que Lamartine a réussi à peindre l'extérieur de Graziella, ce personnage principal autour duquel tourne l'action, tout en mettant l'accent sur cette correspondance entre le physique et l'âme du personnage.

\section{Graziella et les trois éléments de I'univers : -}

Nous découvrons, d'après la lecture de Graziella que, Lamartine $y$ peint trois éléments qu'il croit exister dans l'univers : la nature, l'amour et la croyance religieuse.

En effet, ce sont les trois points qui agissent d'une grande puissance sur l'esprit de l'auteur comme sur son cœur. "Nous avions rencontré la note qui vibre à l'unisson dans l'âme de tous les hommes, de tous les âges et de toutes les conditions, la note sensible, la note universelle, celle qui renferme dans un seul son l'éternelle vérité de l'art : la nature, l'amour et Dieu" ${ }^{38}$.

Voilà pourquoi nous allons expliquer chacun de ces éléments à part et nous allons essayer d'incarner la portée et 
I'importance de chacun de ces trois thèmes sur l'esprit, l'âme et le cœur de l'auteur dans Graziella, puisque l'on peut trouver que ce sont toujours les trois éléments qui dominent toute l'œuvre de Lamartine.

\section{La nature :-}

$A$ vrai dire, la nature se montre un thème bien important chez Lamartine, ce "fils du riche sol" ${ }^{39}$.

La nature était pour lui comme une mère. II avait grandi au milieu d'elle. Le poète lui-même dit: "J'étais né dans les champs; mes premiers spectacles avaient été les ombres des bois, les lits des ruisseaux..." 40 .

Aussi la famille de Lamartine, qui vivait à la campagne, avait-elle une grande influence sur lui : elle oriente son esprit vers la nature qui l'entoure.

C'est ainsi qu'il y avait une relation étroite entre la nature et l'enfant Lamartine depuis son enfance.

Dans Graziella, Lamartine fait appel, à chaque instant, à ce thème tout en parlant des impressions de son enfance : "Nous avions beaucoup vécu, dans notre enfance, de la vie du paysan" ${ }^{41}$.

Aussi peut-on dire que l'éducation qu'il recevait de sa mère jouait notamment un grand rôle à former son imagination et 
sa conception de la nature. Et c'est grâce à sa mère qu'il devient amoureux de la nature; et l'auteur le déclare ouvertement en disant: "Elle me traduisait tout, nature, sentiment, sensation, pensées" ${ }^{42}$.

L'influence de cette conception maternelle était si profonde sur l'esprit de Lamartine que presque tous les thèmes développés dans ses vers, son amour pour la nature, ne seront que l'éclosion des tendres sentiments greffés par sa mère sur son âme.

On peut dire aussi que Lamartine a su une nature vraie et diverse à Milly. II recevait, dans cette nature, une vive profondeur de sentiments naturels en compagnie de petits paysans du village dont il partage les jeux. Dans la formation intellectuelle du poète, ce premier contact direct et quotidien avec la nature est sans doute l'élément le plus important et le plus caractéristique.

C'est ainsi que l'on peut trouver que tout cela a bien préparé Lamartine à recevoir la nature italienne la plus charmante. Le voyage en Italie fait connaître au poète un univers brillant; il lui découvrit une nature nouvelle plus séduisante et plus brûlante que celle de sa Bourgogne. C'est pourquoi, on peut dire que Lamartine part pour l'Italie "avec 
l'enthousiasme d'un enfant qui va voir se lever le rideau des plus splendides scènes de la nature et de la vie" ${ }^{43}$.

II savoure cette nature qui ravissait son esprit et qui le faisait vivre "dans un état constant d'ivresse pendant les longs jours d'attente qui précédèrent le départ" ${ }^{44}$.

Cette nature a une grande puissance sur l'esprit de notre écrivain et sur son cœur ; "ce délire, renouvelé chaque jour par les magnificences de la nature en Savoie, en Suisse, sur le lac de Genève, sur les glaciers du Simplon, au lac de Côme, à Milan et à Florence, ne retomba qu'à mon retour" 45 .

On peut dire aussi qu'il y avait une harmonie complète entre son âme et cette nature italienne. Séduit par les beaux sites et les grands aspects, le poète rêve "le matin, sous les pins aux larges dômes du Monte Pincio; le soir sous les grandes ombres des colonnades de Saint-Pierre; au clair de lune, dans l'enceinte muette de colisee" ${ }^{46}$.

Ainsi, on trouve que cette nature est pour lui le témoin de sa joie ; par sa beauté, elle exalte le bonheur et glorifie le rêve.

Dans Graziella, on trouve aussi que Lamartine se montre un grand contemplateur et trouve à Rome le cadre qui convient le mieux à son esprit comme le montre cette phrase : "J'étudiai 
Rome, son histoire et ses monuments (...) Je faisais de Rome une étude sérieuse, mais une étude en action" ${ }^{47}$.

Après s'être assouvi de Rome, qui "achève de ses ruines, comme de sa grandeur, la mélancolie passionnée de ses songes, le poète voulait à voir Naples où il a trouvé ces paysages dont rien ne peut donner une idée dans aucun autre pays du monde" 48 .

A Naples, c'est le tombeau de Virgile et le berceau du Tasse qui s'emparaient de son esprit et attiraient son imagination.

Le contact avec cette nature nouvelle ranime les sensations du poète. La vue même de ces monuments italiens le ravissait. Charmé par la lueur de la maison du Tasse, il dit : "La lueur de cette maison brillait jusqu'au fond de mon âme. C'était comme un éclair de gloire qui étincelait du loin sur ma jeunesse et dans mon obscurité" 49 .

Lamartine se montre aussi bien sensible de jeux de lumière. En admirant les torches de résine que les pêcheurs allument pour tromper le poisson, il dit: "Ces feux, rouges comme des foyers de fournaise, se reflètent en longs sillons ondoyants sur la nappe de la mer, comme les longues trainées de lueurs qu'y projette le globe de la lune" ${ }^{50}$. 
Lamartine ne se montre pas seulement amoureux de la nature, mais aussi un contemplateur qui veut découvrir les choses mystérieuses de cette nature : "Ces nuits tièdes et lumineuses passées sous la voile, dans un berceau ondoyant des lames et sous le ciel profond et étoilé, nous semblaient une des plus mystérieuses voluptés de la nature, qu'il fallait surprendre et connaître, ne fût-ce que pour la raconter" ${ }^{\prime 51}$.

Aussi remarque-t-on que Lamartine aime les hautes montagnes et s'étonne de l'orgueil des cimes. Le poète qui a tant aimé la charmante île d'Ischia, voit qu'elle "n'est qu'une seule montagne à pic dont la cime blanche et foudroyée plonge ses dents ébréchées dans le ciel ${ }^{52}$.

De même, Lamartine, ce poète rêveur, voit que "les songes ne se développent que dans un milieu liqiuide" ${ }^{53}$. ॥ sent aussi la consonance de son âme avec les bruits maritimes. Il partageait les travaux et les jours du pêcheur, Andréa. Sur la nappe de la mer, le poète dit: "Nous écoutons avec ravissement les gouttes sonores de l'eau, qui ruisselait de nos rames, tomber harmonieusement dans la mer comme des perles dans un bassin d'argent" ${ }^{\prime 54}$. 
Aussi peut-on remarquer que les maisons rustiques sont les lieux qui conviennent le mieux à l'état sentimental de Lamartine. II dit: "Il n'y a pas une de ces maisons suspendues aux pentes de la montagne, cachée au fond de ses ravins, (...) qui ne fût en songe la demeure idéale d'un poète ou d'un amant" ${ }^{55}$.

C'est ainsi que cette nature napolitaine fait le poète vivre dans un état d'ivresse et de délire. Elle a fasciné son esprit par sa beauté et sa puissance extraordinaires. Dans une lettre à son ami Virieu, qui témoigne son ravissement de cette nature pleine du charme, notre auteur dit: "Sais-tu que dans ma belle indifférence, j'étais tenté de ne pas venir à Naples ; j'aurais perdu le plus beau spectacle du monde entier qui ne sortira plus de mon imagination (...) Les mots me manqueraient pour te décrire cette ville enchantée, ce golfe, ces paysages, ces montagnes uniques sur la terre, cet horizon, ce ciel, ces teintes merveilleuses" ${ }^{56}$.

C'est ainsi que l'auteur trouve tout son bonheur et toute sa jouissance au sein de cette nature.

Aussi Lamartine voit-il que la nature est animée. II dit qu'elle "parle la même langue à ceux qui cohabitent avec elle sur la montagne ou sur la mer" 57 . 
Pour lui, la nature a une grande influence sur les hommes. Elle leur donne une simplicité et une certaine pureté. En trouvant toute la bonté parmi la famille du pêcheur qui le traitait comme un frère, Lamartine dit : " Les cœurs s'ouvrent sans défiance, ils se soudent tout de suite, parce qu'il n'y a pas d'intérêt soupçonné sous les sentiments. II se forme plus de liaison et de parenté d'âme en huit jours parmi les hommes de la nature qu'en dix ans parmi les hommes de la société" 58 .

La nature italienne adoucit les souffrances de Lamartine et apaise les fièvres de son âme. En trouvant tout son repos et toute sa détente au milieu de cette vie naturelle, il dit que cette vie, où rien ne tremblait la tranquillité de l'âme, le "plaisait et endormait en lui ces mouvements fiévreux de l'âme, qui usent inutilement l'imagination des jeunes hommes avant I'heure où leur destinée les appelle à agir ou à penser" ${ }^{59}$.

En effet, la sensibilité du poète a été profondément marquée par cette charmante existence de l'Italie. Sa formation spirituelle et sentimentale, sous l'influence de cette nature italienne, laisse une trace naturelle profonde dans Graziella. La beauté de cette nature, que le lecteur savoure sur les pages du roman, ravit son esprit et le fait vivre dans un état d'ivresse. 
Lamartine lui-même dit, dans ses Mémoires inédits, que Graziella "a encore tant de succès parce que tout le monde y a reconnu l'accent réel de la nature" ${ }^{60}$.

\section{L'amour :-}

L'amour est aussi l'un des thèmes fondamentaux dans Graziella de Lamartine.

D'après ce roman, on peut dégager les traits essentiels de l'amour adolescent de Lamartine.

En effet, avant "Graziella", Lamartine, qui essaie de se rendre le plus amoureux possible, a vécu plus d'une aventure d'un amour simple.

A Milly, il rencontre Janette qu'il se voit bien aimer. Elle est "une jeune femme de dix-neuf à vingt ans, très jolie, très bonne, très simple et très naïve qui l'aime" ${ }^{61}$.

Mais Lamartine cherche toujours un amour nouveau. II rencontre, au village de Salongny, près de Milly, une fille de seize ans comme lui. Elle était "musicienne et sa voix faisait pleurer $^{\prime \prime 2}$.

Mais la mère de Lamartine, qui veillait jalousement sur l'âme de son fils, fait partir son fils avant que cet amour ne devienne plus sérieux. 
De même, pendant son séjour à Lyon, le jeune Lamartine échangeait des vers avec Caroline Pascal, fille d'un médecin ; et l'amour est né "sous le couvert de la poésie"63. Mais les parents de Lamartine ont condamné cet amour en disant : "un garçon de dix-neuf ans, la fille d'un médecin, impossible ! "64.

Quelque temps après, il a rencontré Henriette Pommier, la fille d'un honorable juge de paix, et la passion l'a vite dominé. Lamartine sent qu'il l'aime sans mesure. Mais, encore une fois, les Lamartine ont éloigné le fils amoureux. Cela a réussi à calmer la passion ardente de Lamartine et a tempéré ses élans.

C'est pour cela que l'on peut dire que le cœur du futur poète n'a aucun triomphe pendant son adolescence. II ne s'attachait pas d'un amour vrai à aucune de ces femmes qu'il se croyait aimer. Aucune d'elles ne lui paraît digne d'une passion.

Mais avec "Graziella", Lamartine a trouvé les apaisements de son âme. II cherche le bonheur dans l'amour pour que son âme soit satisfaite. C'est l'amour pur et sans tâche qui donne ce bonheur à notre poète. 
En échangeant un regard, un mot amical, un sourire avec Graziella, Lamartine se sentait heureux "jusqu'au fond de l'ame" ${ }^{65}$, Graziella est, pour lui, "la douceur de vivre" ${ }^{66}$.

Lamartine nous montre que l'amour agit d'une puissance sur l'esprit et sur l'âme de l'amant. Graziella le forçait de sortir de sa solitude ; et lui, il n'avait qu'à obéir "en murmurant, mais en l'aimant" ${ }^{67}$.

Mais il voit aussi que ce n'était pas de l'amour vrai ; il résultait de l'inquiétude et du trouble dont il souffrait après le départ de son ami Virieu. Tout ce qu'il a trouvé, c'est le calme de ses sentiments et le repos de son cœur. II ne pouvait préciser son sentiment envers Graziella. II dit : "Je ne savais pas comment je l'aimais ; si c'était de l'intimité pure, de l'amitié, de l'amour, de l'habitude ou de tous ces sentiments réunis que se composait mon inclination pour elle" ${ }^{\prime \prime 8}$.

Menacé de perdre Graziella, il dit encore : "j’éprouvais quelque chose de l'infini de l'amour par l'infini de la tristesse dans laquelle mon cœur se sentit tout à coup submergé" 69 .

Parmi la nature qui a tout enchanté son cœur, qui a ravi son esprit et qui a si bien donné de l'harmonie à son âme, il 
nous montre que l'amour de Graziella l'occupait davantage en disant: "Je regardais tout, je ne vis rien ... Mon âme était ailleurs ; je voulais en vain la rappeler ${ }^{\prime 70}$.

Dans ce roman, on trouve aussi que Lamartine insiste sur l'importance et la nécessité de l'amour dans la vie. Sans l'amour, on ne peut avoir ni le repos, ni l'harmonie. "Ôtez de la vie le cœur qui vous aime : qu'y reste-t-il ? "71.

On trouve aussi, dans "Graziella", un trait essentiel de cet amoureux romantique : il éprouve souvent le désespoir dans son amour. Pour lui, l'amour peut conduire "à la mélancolie mais jamais au ciel noir de l'ennui" ${ }^{72}$.

Pour nous montrer sa douleur extraordinaire après la perte de Graziella, le poète dit : "Un nuage sur l'âme couvre et décolore plus la terre qu'un nuage sur I'horizon" ${ }^{73}$.

Graziella représentait tout pour lui ; quand il a perdu sa bien-aimée, il déclare : "tout me manquait. Je me manquais à moi-même ${ }^{174}$.

D'autre part, on constate qu'il suffit à notre auteur une larme pour que son cœur se vibre et touche l'orage de l'amour. "Je connus l'amour: par une larme dans des yeux d'enfant" 75 . 
Aussi comprend-on que l'amour de Lamartine était incomplet pendant sa jeunesse ; son sentiment d'amour n'était pas sincère ; et il en dit : "Hélas ! ce n'était pas le complet amour : ce n'en était en moi que l'ombre"76 ; "I'homme trop jeune est incapable d'aimer" ${ }^{77}$.

Mais dans une autre étape plus stable de sa vie, Lamartine nous montre l'amour vrai qui s'attache étroitement à la vie, aux expériences acquises. II voit que le véritable amour est "le fruit mûr de la evi"78.

II distingue entre deux sortes d'amour: l'amour adolescent et l'amour complet. Pour nous montrer la différence entre ces deux genres d'amour, Lamartine dit : "Il y a plus de sève folle et d'ombre flottante dans les jeunes plants de la forêt ; il y a plus de feu dans le vieux cœur du chêne" ${ }^{79}$.

C'est pour cela que l'on trouve que le poète ne s'est pas attaché, pendant sa jeunesse d'une manière sincère et sérieuse, à aucune des femmes qu'il a déjà connues. Il les considère seulement comme une étape passagère de sa vie amoureuse.

Dans "Graziella", on trouve aussi que l'absence produit, sur l'imagination du poète, son effet ordinaire et exalte l'amour de l'amant solitaire. Après son départ, il dit : "Graziella n'était pas oubliée, mais elle était violée dans ma vie. Cet amour 
qui enchantait mon cœur, humiliait mon respect humain. Son souvenir, que je nourrissais seulement en moi dans la solitude, dans le monde me poursuivait presque comme un remords" 80 .

Aussi Lamartine montre-t-il, dans son roman, l'importance de la mémoire dans la vie du poète. C'est vrai qu'il n'a pas aimé Graziella d'un amour vrai dans la première étape de son âge, mais il a gardé au moins les plus nobles sentiments envers celle qui a pu incarner une partie de sa vie et de sa mémoire. Après avoir conçu la nouvelle de la mort de la Graziella, Lamartine, qui sentait "une ombre de sa mort répandit dès ce jour-là sur (son) visage et sur (sa) jeunesse" 81 , dit: "le temps efface vite sur la terre, mais il n'efface jamais les traces d'un premier amour dans le cœur qu'il a traversé. Pauvre Graziella !... J'ai aimé ; j'ai été aimé. D'autres rayons de beauté et de tendresse ont illuminé ma sombre route... Mais rien n'a terni ta première apparition dans mon cœur. Plus j'ai vécu, plus je suis rapproché de toi par la pensée" ${ }^{\prime 82}$.

Aussi peut-on remarquer que le poète n'a d'autre mémoire que celle de ses émotions, celle de sa sensibilité et de son imagination, ces larmes qu'il ne sait plus d'où elles lui 
viennent. "Il y a toujours au fond de mon cœur une larme qui ne filtre goutte à goutte et qui filtre en secret sur ta mémoire pour la fraîchir et pour l'embaumer en moi" ${ }^{83}$.

Ainsi on peut voir que cet amour, suivi d'une séparation douloureuse, à cause de la mort de Graziella, conduit Lamartine à sa mélancolie: "mes larmes se séchèrent; mais les nuages, qui avaient traversé ma pensée pendant cette tristesse d'une sépulture, ne s'évanouirent pas ${ }^{84}$.

Pour distraire son "désespoir, fruit naturel de l'arbre de la solitude ${ }^{85}$, et pour tromper son ennui, notre poète compose des vers "intitulés le Premier Regret" ${ }^{86}$.

"Remontez, remontez à ces heures passées !

Vos tristes souvenirs m'aident à soupirer !

Allez où va mon âme ! allez, ô mes pensées !

Mon cœur est plein, je veux pleurer ! ${ }^{" 87}$.

C'est ainsi que l'on trouve que l'amour et la mort de "cette poétique Graziella" ${ }^{88}$ suscitent chez Lamartine les plus vrais, les plus profonds sentiments humains.

\section{La religion :-}

La pensée religieuse de Lamartine est l'un des piliers sur lesquels ce roman est basé. En effet, Lamartine doit sa foi à sa mère dès son enfance. Elle lui a donné un enseignement 
souvent religieux; et c'est grâce à la foi stable de cette tendre mère que la religion s'étend à l'âme de notre auteur. Lamartine lui-même nous révèle cette influence maternelle en disant : "Elle me mettait le doigt sur toutes choses. L'instruction insensible que je recevais n'était pas une leçon, c'était l'action même de vivre, de penser et de sentir que j'accomplissais sous ses yeux, avec elle, comme elle et par elle" ${ }^{\prime 89}$.

D'ailleurs, Lamartine a eu une autre influence bien profonde. II a fréquenté une famille pieuse, celle du peintre romain chez elle, il avait logé. Ce peintre ne sortait jamais de son atelier que pour aller à la messe avec sa femme et sa fille. Quand le soir venait le seul délassement de cette famille "était de dire ensemble le chapelet et de psalmodier à demi-chant les litanies" $"$.

Ayant vécu cette scène calme et pieuse dans la maison de ce peintre, Lamartine se souvient toujours de ce qu'il voyait dans le foyer paternel : "En retrouvant les mêmes habitudes, les mêmes actes, la même religion, je me sentais presque sous le toit paternel dans cette famille inconnue" ${ }^{91}$.

Dans "Graziella", on peut trouver que cette foi héritée de sa mère et cette fréquentation des hommes religieux font naître 
dans le cœur de Lamartine une pitié, une miséricorde et une tendresse. Le poète, qui abhorrait toutes les tyrannies et les servitudes, dit: "d'un son chretien"92, "combien de fois ne versâmes-nous pas des larmes amères sur le sort de ce monde livré à toutes les tyrannies, où la philosophie et la liberté n'avaient semblé vouloir renaître un moment en France et en Italie que pour être souillées, trahies ou opprimées partout! Que d'imprécations à voix basse ne sortaient pas de nos poitrines contre ce tyran de l'esprit humain, ..." ${ }^{93}$.

De même, on trouve que "Le Génie du Christianisme lui impose l'antithèse classique entre Saint-Pierre et le colisée" ${ }^{194}$ : "Le colisée, cet ouvrage du peuple...; SaintPierre, ce chef-d'œuvre du catholicisme" ${ }^{\prime 95}$.

Mais on peut dire aussi que la foi de Lamartine ne s'appuyait sur aucun dogme stable. II refuse d'accepter les croyances de son enfance. II essaye de les sentir et d'y penser ; et tout au long de sa vie, il "a cherché Dieu sans jamais trouver le repos" ${ }^{96}$.

C'est pour cela que l'on peut dire que Lamartine se débat dans le doute tout en essayant de trouver la vérité. II se sentait attiré au rationalisme. 
Aussi sa façon de parler de l'Eglise Saint-Pierre explique-t-elle ce rationalisme: "Les architectes des cathédrales gothiques étaient des barbares sublimes. Michel-Ange seul a été un philosophe dans sa conception. Saint-Pierre, c'est le christianisme philosophique, d'où l'architecte chasse les ténèbres, et où il fait entrer l'espace, la beauté, la symétrie, la lumière à flots intarissables" ${ }^{97}$.

Aussi peut-on dire que ce rationalisme conduit Lamartine au scepticisme. II arrive à dire que le christianisme n'est pas une religion éternelle. "Le christianisme périrait que SaintPierre resterait encore le temple universel, éternel, rationnel, de la religion quelconque qui succèderait au culte du Christ, pourvu que cette religion fût digne de l'humanité et de Dieu" ${ }^{\prime 98}$.

En ajoutant quelques détails dans sa description de Saint-Pierre de Rome, Lamartine nous révèle son caractère trop rationaliste qui veut communiquer avec Dieu dans la lumière. "Saint-Pierre est ... un grand symbole de ce christianisme éternel qui, possédant en germe dans sa morale et dans sa sainteté les développements successifs de la pensée religieuse de tous les siècles et de tous les hommes, s'ouvre à la raison à mesure que Dieu la fait luire, 
communique avec Dieu dans la lumière, s'élargit et s'élève aux proportions de l'esprit humain grandissant sans cesse et recueillant tous les peuples dans l'unité d'adoration, fait de toutes les formes divines, un seul Dieu, de toutes les fois un seul culte, de tous les peuples une seule humanité" 99 .

C'est ainsi que Lamartine est toujours tiraillé entre la tradition familiale, c'est-à-dire la voix du cœur, et les sollicitations de sa raison.

Aussi Lamartine ne s'arrête-t-il pas de chercher le vrai Dieu. Dans son Voyage en Orient, la religion musulmane lui paraît beaucoup plus rationnelle que le christianisme; la mosquée vaut mieux que l'église puisqu'elle n'est pas un temple où habite un Dieu, mais une maison de prière et de contemplation "où les hommes se ressemblent pour adorer le Dieu unique et universel"100. II voit aussi que la pensée de I'Islam est "plus avancée sur la route de la perfection religieuse que beaucoup de religions qui l'insultent et la méconnaissent" ${ }^{101}$.

Mais malgré son scepticisme à l'égard du christianisme, Lamartine était un chrétien soumis car la religion positive est en lui une chose de volonté et de raison plus que de sentiment. 
Aussi Lamartine n'aime-t-il pas les païens ; il laissa la grand-mère de Graziella dire à Andréa : "Ne savais-tu pas que ce sont des païens et qu'ils portent le malheur et l'impiété avec eux ?"102.

En somme, ce roman nous montre que Lamartine cherche toujours Dieu sans trouver ni la paix de l'âme, ni l'accord de son esprit avec son cœur. II n'avait pas un dogme stable car sa religion, libre et vague, paraît moins une fois qu'un sentiment.

\section{Conclusion}

Nous ne pouvons pas nier, à la fin de notre modeste étude, l'importance des aspects stylistiques dans ce roman écrit par un grand poète et un écrivain distingué du $\mathrm{XIX}^{\mathrm{e}}$ siècle par la variété du style. Disons tout d'abord que pour éviter toute séchasse et toute monotonie, l'auteur veille toujours varier son style. Sans entrer dans des détails linguistiques qui méritent une autre étude, signalons que Lamartine a recours à divers procédés pour exprimer ses idées ou même sa sensibilité.

Tout au long de Graziella, il emploie le discours direct pour mettre plus vivement, la scène sous les yeux, pour exprimer avec plus de force la vivacité d'un mouvement de l'âme. Et pour faire nettement sentir le ton du discours, la 
grand-mère de Graziella parle en se lamentant à Andrea et lui dit :

"qu'avais-tu à faire, (...) de prendre ces deux étrangers,

ces deux français avec toi ? Ne savais-tu pas que

ce sont des païens (pagani) "103.

Aussi, après avoir perdu la barque sa seule richesse, elle parle à la mer en gémissant :

"Pourquoi ne nous as-tu pas pris nous-mêmes ? nous tous ? puisque tu nous as pris notre gagne-pain ? Tiens! tiens! tiens! prends-moi du moins en Moreaux, puisque tu ne $m$ 'as prise toute entière" ${ }^{104}$.

Pour abréger, Lamartine emploie dans Graziella le discours direct qui lui paraît plus rapide, en particulier dans une longue conversation entre deux ou plusieurs personnages comme, par exemple, entre le pêcheur, Lamartine et son ami.

C'est ainsi qu'on peut trouver que le discours direct est utile à l'intérêt du roman et à faire connaître le personnage.

Cependant, de temps en temps, pour éviter la monotonie, Lamartine utilise dans Graziella le discours indirect qui sert à exprimer les réflexions d'un personnage : 
"Graziella nous demandait souvent qu'est-ce que nous lisions donc tout le jour dans nos livres" 105.

Ajoutons que Lamartine, dans Graziella, a recours à des phrases interrogatives pour mettre en valeur une idée ou pour attirer l'attention du lecteur sur ce qu'il dit :

"La nature elle-même (...) qu'a-t-elle autre chose que deux ou trois paroles et deux ou trois notes, toujours les mêmes, ...." ${ }^{106}$.

Nous remarquons aussi, dans Graziella que Lamartine, pour mettre quelques idées en relief et transmettre sa réaction personnelle au lecteur, emploie des phrases exclamatives :

"Combien le vent et les vagues de la mer de Naples n'ont-ils pas emporté et englouti, le matin, de Lambeaux de mes sentiments et de mes pensées de la nuit, déchirés le jours et s'envolant sans regret loin de moi" 107 .

Nous pouvons voir que l'auteur de Graziella s'y adresse au lecteur d'un loin impératif pour l'attirer a son idée :

"changez le prêtre, ôtez l'autel, détachez les tableaux, emportez les statues, rien n'est changé, c'est toujours la maison de Dieu $! . . .{ }^{108}$. 
C'est ainsi que Lamartine est soucieux de la variété. II varie le tour de sa langue et réussit à entrainer le lecteur par le choix du sujet sentimental de son roman Graziella et par son style attirant sans ennuyer ni fatiguer le lecteur avide de lire ce genre de romans romantiques intéressants. Cependant que Graziella montre plusieurs autres aspects de l'écrire ou de l'éloquence de Lamartine. Nous avons tenu simplement à signaler quelques aspects saillants qui pourraient mettre en valeur Graziella riche, de thèmes et digne d'être analysé avec plus de profondeur et d'intérêt soit dans son fond et dans sa forme. 


\section{$\underline{\text { Marge }}$}

1. Toesca (Maurice), "Lamartine ou l'amour de la vie", Paris, Albin Michel, 1969, P. 466.

2. C.F. "Graziella, un roman expérimental ?" in http://www.lavisiteaumusee.fr, 11 décembre 2019

3. De Luppé (Marquis), "Les travaux et les jours d'Alphonse de Lamartine", Paris, Albin Michel, 1942, P.31.

4. Lamartine (Alphonse de), "Graziella", Paris, Raphaël, 1936.

5. De Luppé (Marquis), "Les travaux et les jours d'Alphonse de Lamartine", op.cit., P.33.

6. Duberton (J. J. Lucas), "Lamartine", Paris, Flammarion, 1951, P.9.

7. Ibidem.

8. Lamartine (Alphonse de), "Cours familier de littérature : Un Entretien Par Mois", Paris, Library of Alexandria, 1858, P. 25.

9. Duberton (J. Lucas), "Lamartine", op.cit., P.31.

10. De Luppé (Marquis), "Les travaux et les jours d'Alphonse de Lamartine", op.cit., P.31-32.

11. Duberton (J. Lucas), "Lamartine", op.cit., P.30.

12. De Luppé (Marquis), "Les travaux et les jours d'Alphonse de Lamartine", op.cit., P.34.

13. Lamartine (Alphonse de), "Graziella", P. 64. 
14. Ibid., P. 115.

15. Ibid., P. 84 .

16. Ibid., P. 116.

17. Ibidem.

18. Ibid., P. 124.

19. Ibid., P. 63.

20. Ibid., PP. 63-64.

21. Ibid., P. 64.

22. Viallaneix (Paul), "Lamartine : le livre du centenaire", Paris, Flammarion, 1971, P.40.

23. Lamartine (Alphonse de), "Graziella", P. 111.

24. Ibid., P. 63.

25. Ibidem.

26. Ibid., P. 64.

27. Ibidem.

28. Ibid., P. 100.

29. Ibid., P.101.

30. Ibid., 103.

31. Ibid., 90.

32. Ibid., P. 110.

33. Ibidem.

34. Ibid., P. 126. 
35. Ibid., PP. 124-125.

36. Ibid., P. 125.

37. De Luppé (Marquis), "Les travaux et les jours d'Alphonse de Lamartine", op.cit., P.35.

38. Lamartine (Alphonse de), "Graziella", PP. 96-97.

39. Moreau (Pierre), "Le romantisme", Paris, Del Duca, 1957, P. 132.

40. Boeniger (Yvonne), "Lamartine et le sentiment de la nature", Paris, Nizet, 1934, P. 8.

41. Lamartine (Alphonse de), "Graziella", P. 53.

42. Boeniger (Yvonne), "Lamartine et le sentiment de la nature", op.cit., P. 1.

43. Lamartine (Alphonse de), "Graziella", P. 29.

44. Ibid., P. 30 .

45. Ibidem.

46. Ibid., P. 33.

47. Ibid., P. 38 .

48. Gustave (Frejaville), "Les Médiations de Lamartine", Paris, Sflet, 1947, P. 46.

49. Lamartine (Alphonse de), "Graziella", P. 44.

50. Ibid., P. 46.

51. Ibid., PP. 47-48. 
52. Ibid., P. 51.

53. Viallaneix (Paul), "Lamartine : le livre du centenaire", op.cit., P. 18.

54. Lamartine (Alphonse de), "Graziella", P. 50.

55. Ibid., P. 51.

56. Boeniger (Yvonne), "Lamartine et le sentiment de la nature", op.cit., P. 28.

57. Lamartine (Alphonse de), "Graziella", P. 53.

58. Ibid., P. 115.

59. Ibid., P. 47.

60. Ibid., P. 218.

61. Duberton (J. Lucas), "Lamartine", op.cit., P. 22.

62. Ibid., P. 23.

63. Ibidem.

64. Ibidem.

65. Lamartine (Alphonse de), "Graziella", P. 118

66. Toesca (Maurice), "Lamartine ou l'amour de la vie", op.cit., P. 254.

67. Lamartine (Alphonse de), "Graziella", P. 129.

68. Ibid., P. 140.

69. Ibid., P. 141.

70. Ibid., P. 144. 
71. Ibid., P. 143.

72. Sagnes (Guy), "L’Ennui dans la littérature française de Flaubert à Laforge, 1848 - 1884", Paris, A. Golin, 1969, P. 350.

73. Lamartine (Alphonse de), "Graziella", P. 144.

74. Ibid., P. 145.

75. lbid., P. 166.

76. Ibid., P. 161.

77. Ibid., P. 183.

78. Ibidem.

79. Ibidem.

80. Ibid., P. 182.

81. Ibid., P. 184.

82. Ibid., P. 185.

83. Ibidem.

84. Ibid., P. 186.

85. Toesca (Maurice), "Lamartine ou l'amour de la vie", op.cit., P. 254.

86. Lamartine (Alphonse de), "Graziella", P. 186.

87. Ibid., P. 192.

88. Moreau (Pierre), "Le romantisme", op.cit., P. 133.

89. Gustave (Frejaville), "Les Médiations de Lamartine", op.cit., P. 35. 
90. Ibid., P. 35.

91. Ibidem.

92. Lebey (André), "Lamartine dans ses horizons", Paris, Albin Michel, 1929, P. 36.

93. Lamartine (Alphonse de), "Graziella", P. 37.

94. De Luppé (Marquis), "Les travaux et les jours d'Alphonse de Lamartine", op.cit., P. 43.

95. Lamartine (Alphonse de), "Graziella", P. 39.

96. Guillemin (Henri), "Lamartine", Paris, Seuil, 1987, P. 107.

97. Lamartine (Alphonse de), "Graziella", P. 40.

98. Ibidem.

99. Ibid., P. 41.

100. Guillemin (Henri), "Lamartine", op.cit., P. 124.

101. Ibid., P. 125.

102. Lamartine (Alphonse de), "Graziella", P. 67.

103. Ibid., PP. 67-68.

104. Ibid., P. 71.

105. Ibid., P. 94.

106. Ibid., P. 103.

107. Ibid., P. 110.

108. Ibid., P. 14. 


\section{Bibliographie}

\section{II- Corpus : -}

- Lamartine (Alphonse de), "Graziella", Paris Raphaël, 1936.

\section{III- Ouvrages généraux :-}

- Benichou (Paul), "Les images romantiques", Paris, Gallimard, 1988.

- Deloffre (Frédéric), "Stylistique et poétique françaises", Paris, société d'édition et d'enseignement supérieur, 1970.

- Fontanier (Pierre), "Les figures du discours", Paris, Flammarion, 1977.

- Henriot (Emile), "Les romantiques", Paris, Albin Michel, 1953.

- Moreau (Pierre), "Le romantisme", Paris, Del Duca, 1957.

- Richard (J. Pierre), "Etudes sur le romantisme", Paris, Seuil, 1970.

- Sagnes (Guy), "L’Ennui dans la littérature française de Flaubert à Laforge, 1848 - 1884 de Flaubert à Laforge, 1848 - 1884", Paris, A. Golin, 1969.

- Yves (Le Hir), "Styles", Paris, K. Lincksieck, 1972.

\section{IV- Ouvrages sur Lamartine et Graziella : -}

- Boeniger (Yvonne), "Lamartine et le sentiment de la nature", Paris, Nizet, 1934.

- De Luppé (Marquis), "Les travaux et les jours d'Alphonse de Lamartine", Paris, Albin Michel, 1942. 
- Des Cognets (Jean), "Préface de Graziella", Paris, Garnier, 1924.

- Duberton (J. J. Lucas), "Lamartine", Paris, Flammarion, 1951.

- Gustave (Frejaville), "Les Médiations de Lamartine", Paris, Sflet, 1947.

- Guillemin (Henri), "Lamartine", Paris, Seuil, 1987.

- Lebey (André), "Lamartine dans ses horizons", Paris, Albin Michel, 1929.

- Levaillant (Maurice), "Lamartine et I'Italie en 1820", Paris, Flammarion, 1944.

- Roth (Georges), "Préface de Graziella", Paris, Larousse, 1925.

- Toesca (Maurice), "Lamartine ou l'amour de la vie", Paris, Albin Michel, 1969.

- Viallaneix (Paul), "Lamartine : le livre du centenaire", Paris, Flammarion, 1971.

\section{V- Sites : -}

1. "Graziella, un roman expérimental ?" in http://www.lavisiteaumusee.fr, 11 décembre 2019. 


\section{ملخص}

كان لامارتين شاعر وكاتب نثز وسياسيًا. بعد وفاة والدته، تأثز كثيراً وكان يبحث بقوة عن مكان ملهم يساعده على الخروج من حالة الحزن المسيطرة عليه، لعله يجد فيه السلوى والإلهام. في عام 1 إ) ، قام بأول رحلة له إلى إيطاليا والتي أثرت عليه بشكل كبير • وهناك التقى بجرازيلا، بطلة روايته التي سميت باسمها، وكانت هذه الفتاة مثالية للغاية لتكون ملهمته لكتابة روايته التي كتبها في سن الثالثة والخمسين. يمكننا القول إن جرازيلا رواية من أكثر الاعمال قرباً إلى قلبه ورقياً في أسلوب السرد في النثر كما لو كان يكتب شعراً. في هذه الرواية المميزة، يسعد لامارتين العقل بأسلوبه التصويري الجيد الذي يجعل القارئ في حالة من النشوي والإحساس بالأحداث كما لو كان يعيشها بنفسه. لهذا السبب يمكننا القول إن لامارتين شاعر وكاتب . روائي متميز • تهنم دراستتا بشكل خاص بتحليل شخصية جرازيلا، الثخصية الرئيسية للرواية، وبعد ذلك سوف ندرس العناصر الثلاثة المريمنة في هذه الرواية : الطبيعة والحب والدين. وأخيرًا ، نحاول إلقاء الضوء على التتوع الأسلوبي للروائي لامارتين. 